\title{
Estimation of Extended Plastic Zone in The Ground with The Deformation of Cylindrical Cavity
}

\author{
Satoshi Sugimoto ${ }^{1}$, Hidetoshi Ochiai ${ }^{1}$, Noriyuki Yasufuku ${ }^{1}$, A. Kawamura ${ }^{1}$, Hajime Imanishi ${ }^{2}$ \\ ${ }^{1}$ Department of Civil Engendering, Kyushu University, Fukuoka, Japan \\ ${ }^{2}$ Samsung Corporation, Korea
}

\begin{abstract}
Recently, in Japan, the development of underground utilization in urban area provides a lot of case studies on the excavation of cylindrical cavity (e.g. shield tunnel) in the shallow ground. Especially, in center of the cities, the situation of underground has become complicated, so as to excavate new cavities by neighboring construction. However, the present condition is not clear on the influence caused by such situation. It is important to understand the behavior for making that influence clear. Therefore, the authors try to clarify a mechanism of the earth pressure changes and the ground displacement around the cavity when the section of cavity deforms. It is mainly focused on two factors, namely, the overburden pressure and the extent of the plastic zone caused by the deformation of cavity section. The model tests and theoretical analysis are carried out, taking these factors into consideration. In this paper, it is mainly discussed that the extended plastic zone in the circumference ground with the contraction of cavity is expressed quantitatively and easily by the cavity expansion theory, which introduced the effect of overburden pressure.
\end{abstract}

\section{INTRODUCTION}

Over the past decades, various infrastructures have been constructed in Japan. All sorts of structures have been already congested with the concentration of population and industry at city parts. In future, the further land space will be needed, and the efficient use of underground is required. A lot of structures exist in a relatively shallow underground that has ever been utilized until now. The shallow part of underground can be primarily used for construction when a structure has to be constructed at the both of ground and underground. Then it is hoped that the influence on the existing structure caused by the neighbouring construction is estimated beforehand in such case.

In the case of a cylindrical cavity such as a tunnel, it is considered that one of the factors about the influence is the ground deformation around the cavity with the change of the earth pressure. This behaviour is due to deformation around the cavity. Those causes depend on the constructing method of cavity. From the past time, a lot of studies have been done to estimate the earth pressure on the cavity in such conditions. For example, Culmann's theory which calculated the equilibrium of soil column in the ground, Bierbaumer's theory, which noticed the failure slope with a gradient, and Terzaghi's (1943) theory which examined by trap door test have been developed. Though it is possible to calculate the earth pressure on the cavity by these methods, measuring the distribution of stress and displacement around the cavity seems to be difficult. On the other hand, cavity expansion theory (Yu, 2000) examines the deformation or pressure distribution caused by the expansion or contraction of a cavity. It is expected to obtain the useful information in a case of cavity neighbouring construction.

It is assumed that the plastic zone develops caused by the deformation of cavity section around the cavity. The distribution of ground deformation and earth pressure around the cavity is experimentally 

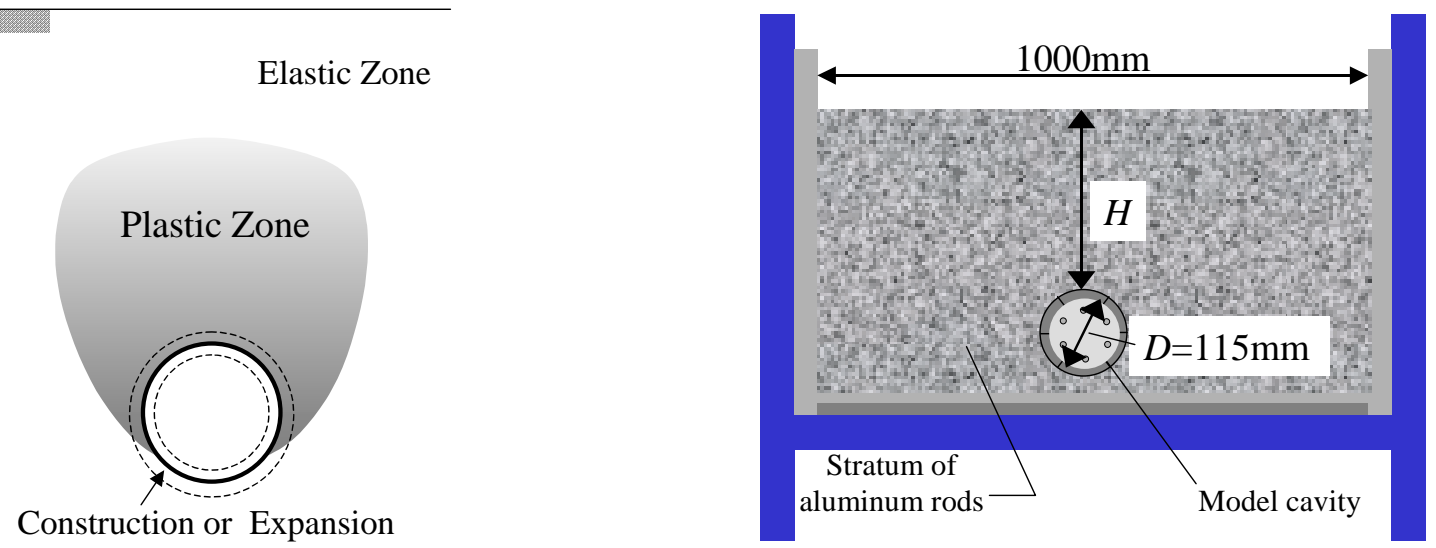

Figure 1. Image of plastic zone around the cavity Figure 2.Test apparatus

investigated in this study. The situation is schematically shown in Figure 1. Therefore, the model tests reproduce the situations that the cylindrical cavity deforms uniformly in radial direction. In addition, the analysis by the cavity expansion theory has been performed to model tests and the applicability of this model is examined.

\section{Experimental observation}

\subsection{Outline of model tests}

In this study, it is supposed that a cylindrical cavity contracts or expands equally in radial direction. The model test is intended for a biaxial plane strain condition because it is assumed that the uniform deformation happens in radial direction of the cavity. The ground behavior caused by cavity deformation can be reproduced by the model test.

The test apparatus is shown in Figure 2. The model ground is made by the aluminum rods with the diameter $\phi=1.0$ and $1.6 \mathrm{~mm}$, whose length is $50 \mathrm{~mm}$. These are mixed with the weight ratio of $3: 2$. The material properties are shown in Table 1. These aluminum rods are laid in the box to a designated height.

Figure 3 shows the new model device developed which can reproduce the deformation of the cavity in the model ground. This is called model cavity. The model cavity has six segments, and the diameter is $115 \mathrm{~mm}$ and the width is $50 \mathrm{~mm}$. A rubber tube is arranged inside this device. Each segments fixed at two points can move in the radial direction by applying the air pressure in this tube. It is possible to make the smooth deformation of the model cavity by introducing a rubber band binding around the segments. The situation of deformation with decompressing the air pressure is shown in Photo 1. The stroke of the segments is $10 \mathrm{~mm}$. This value is so excessive, however it is easier to confirm the failure mode caused by deformation. The cavity deformation is reproduced after setting model cavity in the ground.

Table 1. Material properties

\begin{tabular}{cc}
\hline The weight ratio of aluminum rods & $\phi 1.0 \mathrm{~mm}: 1.6 \mathrm{~mm}=3: 2$ \\
\hline Cohesion & None \\
\hline Friction angle $(\mathrm{deg})$ & 20.0 \\
\hline Density $\left(\mathrm{kN} / \mathrm{m}^{3}\right)$ & 19.6 \\
\hline
\end{tabular}

The experiments have been carried out under various overburden heights $(H=1.0 D, 1.5 D, 2.0 D, 2.5 D$, $3.0 D$ ) for the contracting and the expanding conditions. However, earth pressure tests were only examined under contraction conditions. 


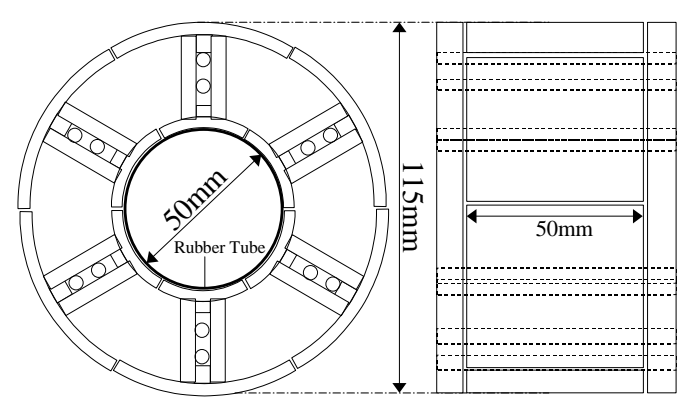

Figure 3. Model cavity

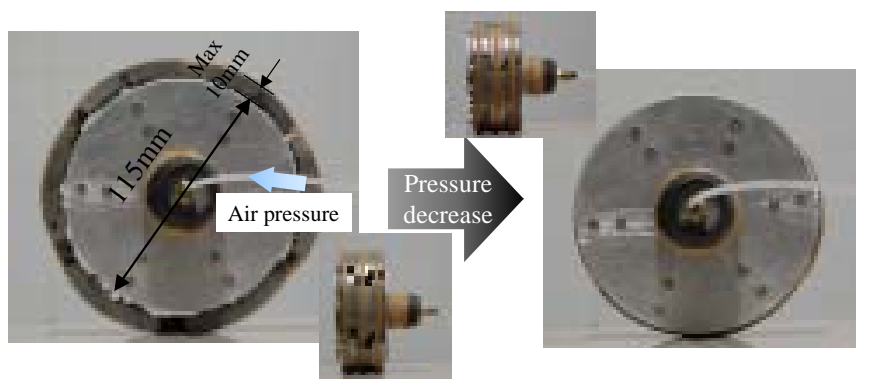

Photo 1. Simulation of the cavity contraction

\subsection{Earth pressure in model ground}

In order to measure the stress in the ground, which is modeled by the aluminum rods, several methods have been tried until now. Two typical methods are used, one uses photo elastic material and another uses load cells at the corner of the model ground. Each of them has some weak points such as the difficulty of quantitative measurement or the measurement at optional locations.

A new device for measuring stress is produced (Kawamura et al., 2003) shown in Figure 4 or Photo 2. This device has a pair of rubber chip at both ends, and it has been very simple structure that a thin rod fixes them. A tri-axial rosette gage is stuck on one of chips. These devices are buried in the model ground. Strain of these three gages is measured at each step before and after the deformation of the cavity. Mohr's strain circle is drawn as shown in Figure 5 by the measured strain of gages. The strain of the optional direction is given by following equations (1)-(3).

The center of Mohr's circle

$$
a=\left(\varepsilon_{a}+\varepsilon_{c}\right) / 2
$$

The radius of the Mohr's circle

$$
r=\frac{1}{2} \sqrt{\left(\varepsilon_{a}-\varepsilon_{c}\right)^{2}+\left\{2 \varepsilon_{b}-\left(\varepsilon_{a}+\varepsilon_{c}\right)\right\}^{2}}
$$

The angle between $\varepsilon_{a}$ and the principal strain

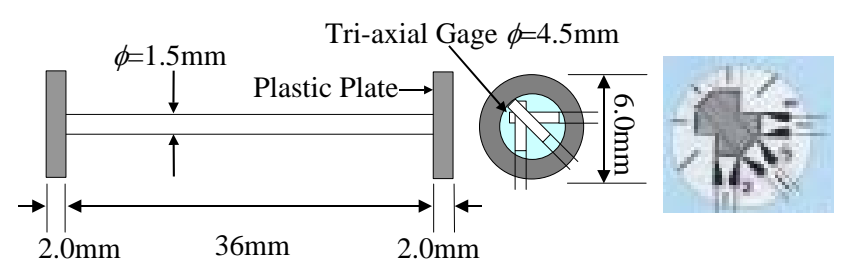

Figure 4. Stress-measuring gage

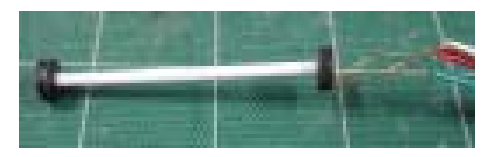

Photo 2. Stress-measuring gage

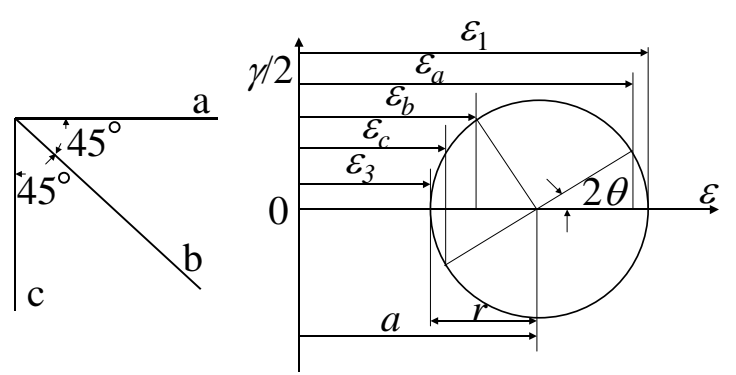

Figure 5. Mohr's circle on the strain 


$$
\tan 2 \theta=\frac{2 \varepsilon_{b}-\left(\varepsilon_{a}+\varepsilon_{c}\right)}{\varepsilon_{a}-\varepsilon_{c}}
$$

Thus, the vertical and horizontal stress on the stress-measuring device is calculated from Equation (4) if assuming the plain stress condition.

$$
\sigma_{h}=\frac{E}{1-v^{2}}\left(\varepsilon_{h}+v \varepsilon_{v}\right) \quad \sigma_{v}=\frac{E}{1-v^{2}}\left(\varepsilon_{v}+v \varepsilon_{h}\right)
$$

The test results in Figure 6 show the stress change around the contracting cavity. Fig. (a) indicates the outline of location between the model cavity and the stress-measuring devices. Figs. (b)-(i) express the stress distributions on each section. It should be noticed that stress values in Figs. (b)-(i) are normalized by the averaged overburden pressure. It can be seen from Figs. (b)-(e) that are the distributions of the normalized vertical earth pressure on the horizontal sections "a" to "d" (see Fig. (a)), it is recognized that the earth pressure increases from the initial stress at the range from 1.0 to 1.5 in horizontal axis. On the other hand, it is characteristic behavior that the horizontal or vertical stress decreases around the cavity. These results show the active or passive condition caused by the contraction of the cavity in the circumference ground. In addition to this, if it is considered that the slip surface is generated nearby the maximum points of the earth-pressure changes, the inside of the line connected with these points can be regarded as the "looseness area". In this study, this area is called the plastic zone as the constant earth-pressure changes can be regarded as getting to the critical state around the cavity.

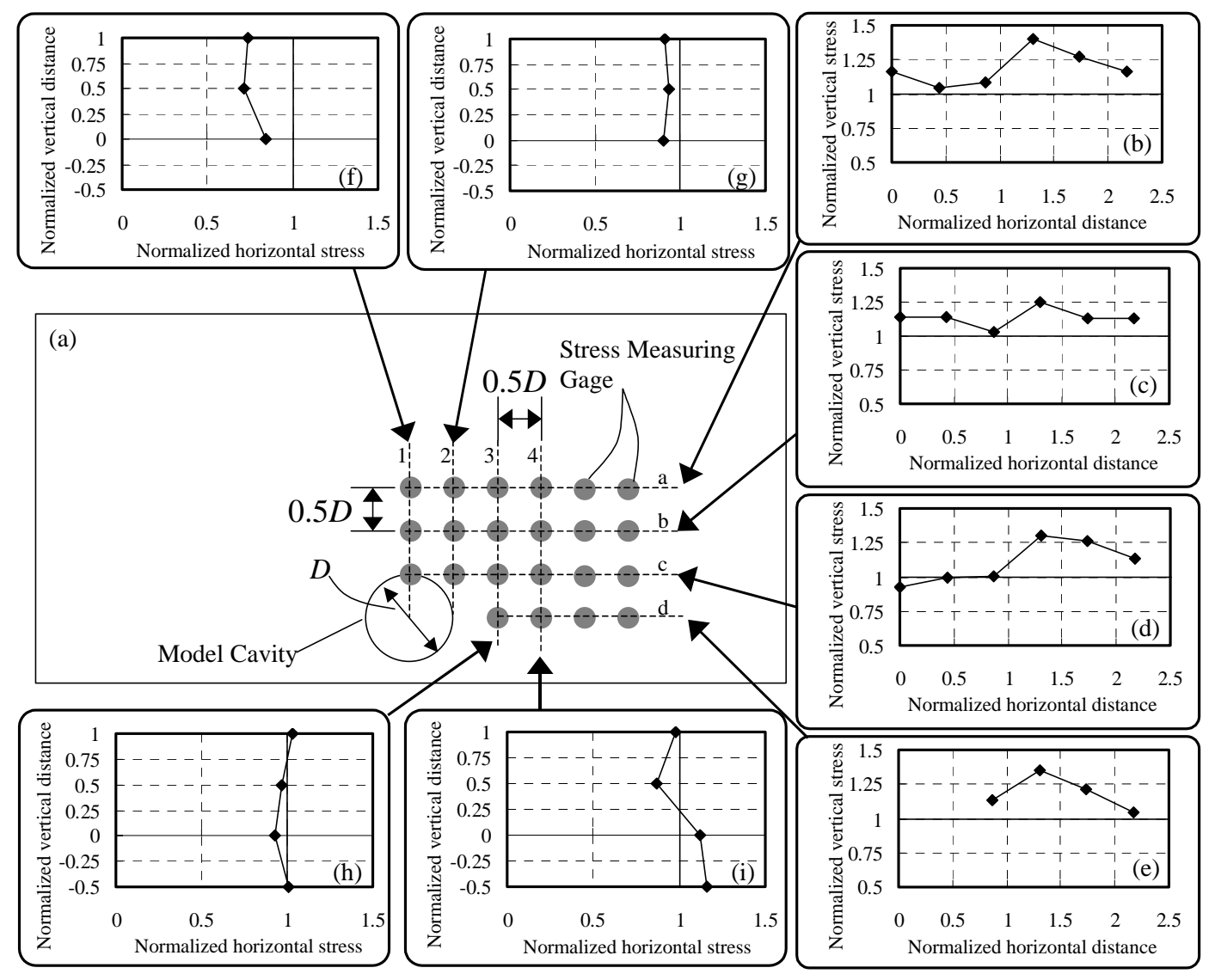

Figure 6. Variation of the earth pressure in the model ground 


\section{APPLICABILITY OF CAVITY EXPANSION THEORY}

\subsection{Application for the contraction of cavity}

Assuming the cavity expansion theory is supposed to cause deformation or stress change symmetrically when the initial stress is an isotropic pressure condition. The logical analysis is tried for a contraction of cylindrical cavity shown in Figure 7. The failure criterion of the ground material is assumed to obey the Mohr-Coulomb's condition.

A large number of information of this theory can be provided from the reference (e.g. Yu, 2000). A typical procedure of calculation on the earth pressure and displacement is shown in this section. Each parameter $a, c$ and $p$ means the radius of the cavity, the radius of the elastic-plastic boundary and the internal pressure of the cavity. Suffices "0" mean initial value. The known quantities are $a_{0}$ and $p_{0}$. The following equations show the displacement $u$ at the elastic zone and the internal pressure of the cavity $p$.

$$
\begin{gathered}
u=r-r_{0}=-\frac{(1-\alpha) p_{0}+Y}{2(1+\alpha) G}\left(\frac{c}{r}\right)^{2} r \\
p=\frac{1}{\alpha-1}\left[-Y+\frac{2\left\{Y+(\alpha-1) p_{0}\right\}}{1+\alpha}\left\{\frac{1-\left(\frac{a_{0}}{a}\right)^{1+\beta}}{1-\left(\frac{c_{0}}{c}\right)^{1+\beta}}\right\}\right.
\end{gathered}
$$

where $Y$ is the Mohr-Coulomb criterion coefficient, $G$ is the shear modulus and $\beta$ is a simple function of dilation angle $\varphi . Y, \beta$ and $\alpha$ take the forms $Y=2 C \cos \phi /(1-\sin \phi), \alpha=(1+\sin \phi) /(1-\sin \phi), \beta=(1+\sin \varphi) /(1-$ $\sin \varphi$ ).

It is possible to determine the stress distribution around the contracting cavity and the size of plastic zone in the case of initial isotropic pressure. However, in this model the influence of an anisotropic overburden pressure in the ground is ignored. In this study, it is, therefore, assumed that the superposition of earth pressure can apply to the calculation. The method of calculation is simple, which is the sum of the original value of cavity expansion theory and the stress of differences from the reference depth.

\subsection{Displacement of the ground around cavity}

Figure 9 shows the relationship between vertical displacements (settlement or heaving) at the ground surface just above the cavity and the overburden height. Each parameter for the vertical and horizontal axes is normalized by the displacement at the top of cavity and the cavity diameter. These parameters

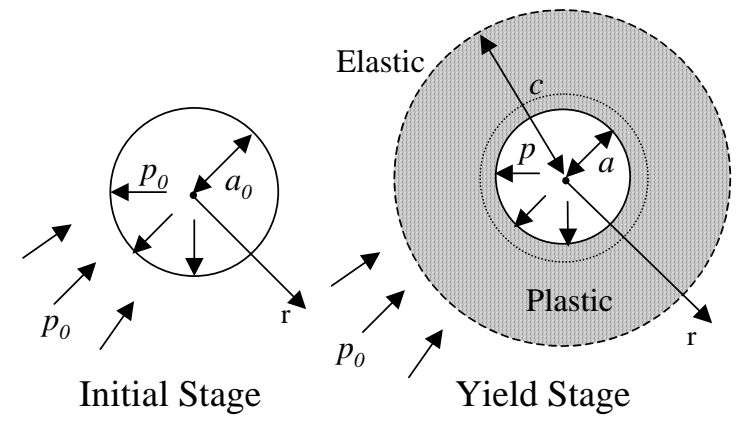

Figure 7. Image of cavity expansion theory

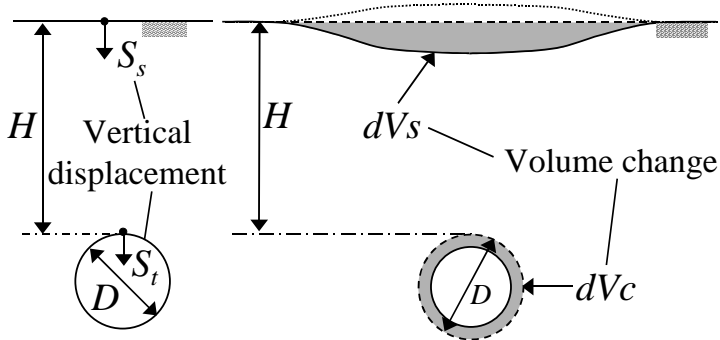

Figure 8. Definition of $S_{s}, S_{t}, d V_{s}, d V_{c}$ 


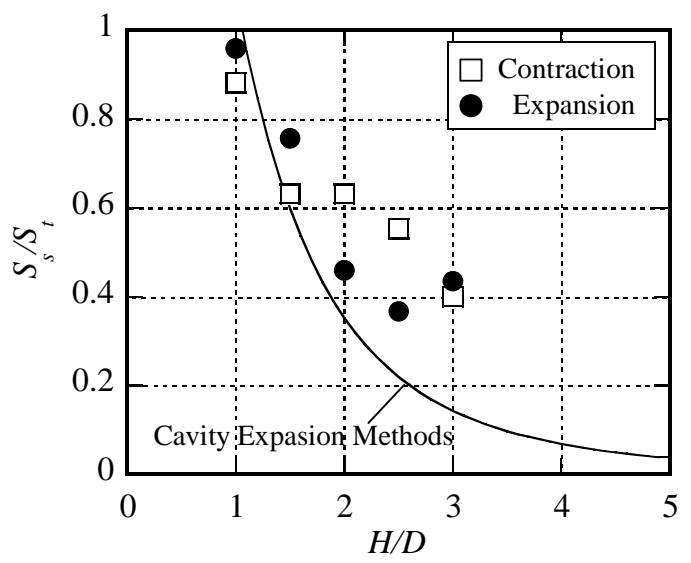

Figure 9. $S_{s} / S_{t}-H / D$

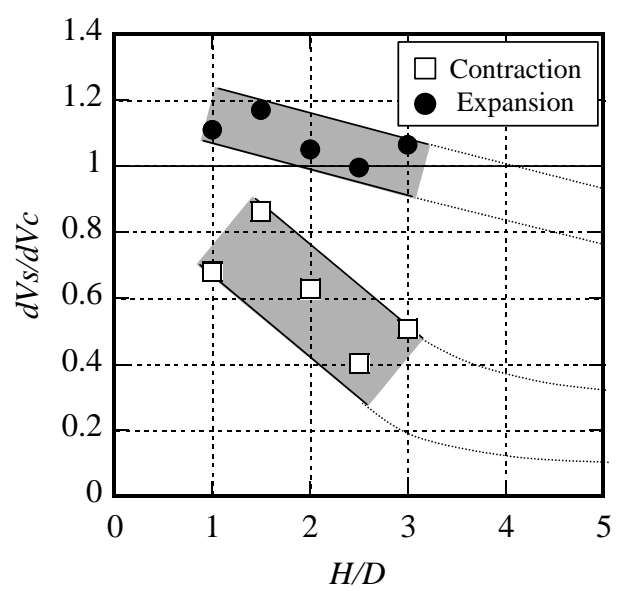

Figure 10. $d V_{s} / d V_{c}-H / D$

are shown in the left side in Figure 8. The plots mean the experimental value and the solid line means the calculated value by cavity expansion theory for contraction. The more $H / D$ increases, the more $S_{s} / S_{t}$ decreases. It is not dependent on the contraction or the expansion. It is, therefore, recognized that the more overburden height increases, the more the influence of cavity deformation extends. $S_{s} / S_{t}$ will converge at around $H / D=5.0$ if the tendency of theory is correct. This value can be the parameter for a distinction between "shallow" or "deep" ground.

The relationship between the volume change and overburden height is shown in Figure 10. $d V_{s}$ and $d V_{c}$ mean the volume change per a unit depth at the ground surface and around the cavity. It can be seen that the determination of the parameters is shown in the right side of Figure 8. The value of $d V_{s} / d V_{c}$ gradually declines with increasing $H / D$. Further; the values of $d V_{s} / d V_{c}$ in the contracting cases are smaller than these in the expanding cases. It can be certified that only a part of total volume change of cavity appears at the level of the ground surface in the contracting cases although the total volume change almost reaches in the expanding cases. It should be, therefore, considered that a part of cavity

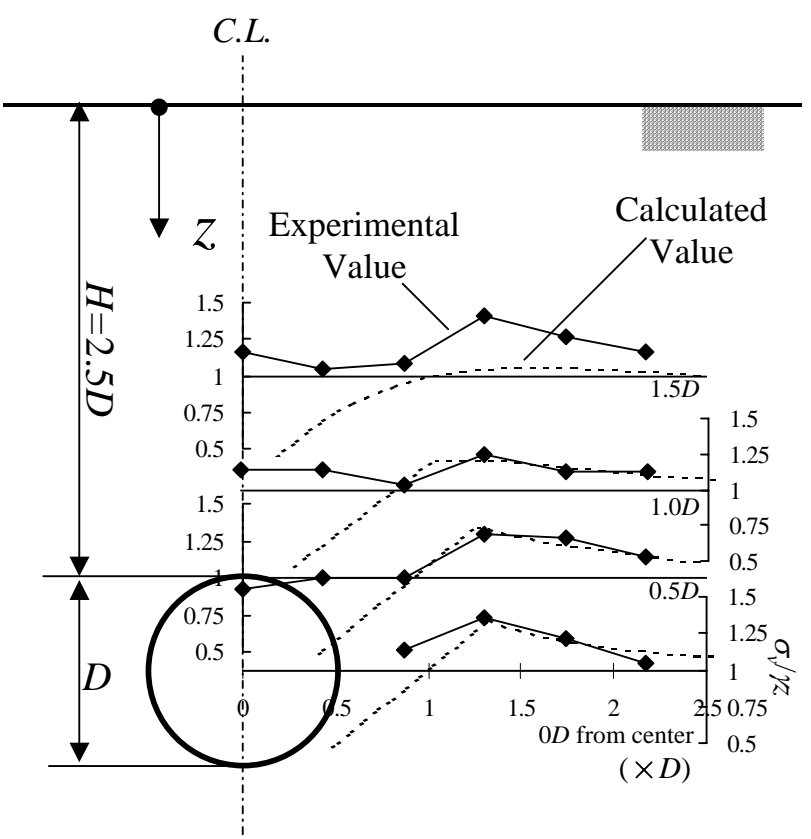

Figure 11. Earth pressure distribution around the cavity volume change is absorbed in the ground between the ground surface and the cavity when the cavity contracts. It is supposed that this behaviour provides the plastic zone in the ground.

\subsection{Earth pressure distribution around the cavity}

The comparison of the experimental earth pressure and those from the cavity expansion theory is shown in Figure 11, in which the solid line with the square plots and the broken line express the results of the experimental value and the calculated value. The horizontal axes signify the geometrical relationship as each of horizontal axes coincides with the location of horizontal section. The value of vertical axes indicates the vertical earth pressure normalized by the overburden pressure at each level. What has to be noticed is the 
situation that the plastic zone doesn't reach the section of $1.5 \mathrm{D}$ from centre of the cavity in the calculation, as the radius of the elastic-plastic boundary is around 1.3D. At a beginning, the calculated value is paid attention to. The earth pressure-increasing zone exists at the location that is $1.0 \mathrm{D}$ or $1.5 \mathrm{D}$ distant in the horizontal direction from the centre of the cavity. The elastic-plastic boundary can be shown at the maximum value of earth pressure. It is considered that the stress decrease caused by the volume expansion reproduces as the earth pressure decrease remarkably nearby the cavity. It is different with both of the experiment and the theory at the surrounding cavity although the stress increasing coincides with them at the location that can be estimated as a slip surface in the ground. Accordingly it can be guessed that the stress-measuring device cannot catch the tendency of stress decrease. However, it is emphasized that the zone where the earth pressure remarkably increases is specified so much when using the presented stress-measuring device.

\section{The vertical-stress distribution in the case of the contraction of twin circular cavities}

In this section, it is noticed the cases that the twin parallel cavities are deformed similarly, and the applicability of the estimation method in the forward section is examined. This "deformation" means the changes of cross-section area, and the mode or the procedure of deformation is not discussed in this paper. All values of the earth pressure in this section are normalized by the initial overburden pressure.

A series of the model tests in the case that two cavities contract were carried out in order to clarify the earth-pressure distribution (Sugimoto et al. 2002). The test box was the same as that in Figure 2. The vertical earth pressure was measured by the load-cells at the bottom of the test box. In this case, the obtained earth pressure means the average of the vertical earth pressure measured at each location of load cells. And the theoretical values are calculated by the presented cavity expansion theory in the third section.

Figure 12 shows the distribution of the vertical earth pressure at the depth that suit the bottom of the test box when one of the cavities was contracted about $7 \%$ of the cross-section area. It can be almost shown the coincidence between test values and theoretical ones. Figure 13 shows the distribution of the vertical earth pressure when the both of cavities were contracted. In which the broken line and the chain line express the values that draw the result of Figure 12 directly corresponded to the location of the each cavity, and the true line shows the total value of those lines. From Figure 13, total-value line shows qualitatively the tendency of test value. And it can be confirmed that the part of bold line corresponds with the test value when the less value are adopted from the combination of the broken line and the chain line. In the sense of the qualitative evaluation, it is possible to specify the zone of the earth-pressure changes by this method. It is, however, necessary to improve this method on the

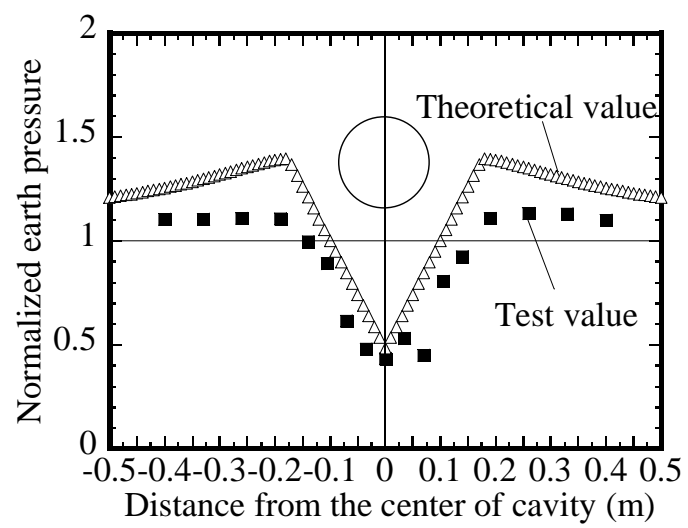

Figure 12.Earth pressure for single cavity

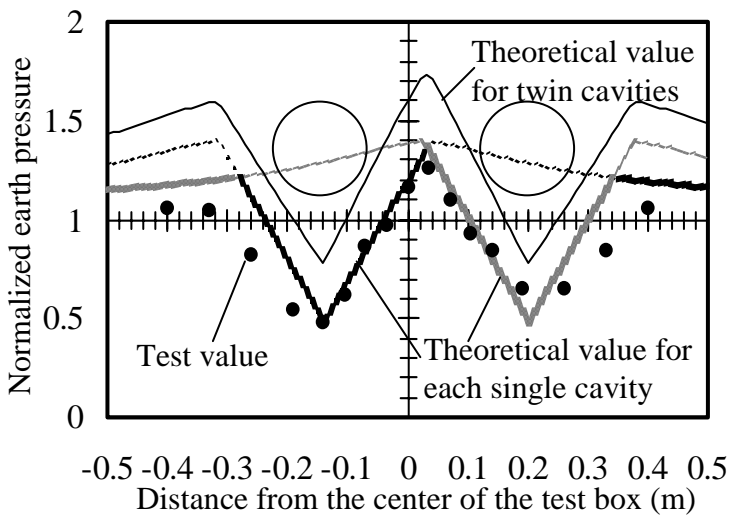

Figure 13.Earth pressure for twin cavities 
way that the effect of the deformation mode, the procedure and the overburden pressure are introduced in the initial condition.

\section{Conclusions}

The two-dimensional model tests were carried out to investigate the ground behavior around the cavity when the isotropic deformation occurs in the section of cavity. In addition, cavity expansion theory was used for searching the possibility of quantitative expression on the ground behavior. The following conclusions were drawn:

1. Regardless of the contraction or expansion of the cavity, the quantitative relation was similar relationship the vertical deformation of ground surface and the overburden. The vertical deformation of the surface just above the top of cavity is almost constant when the overburden height is more than around 5.0 times of the cavity diameter in this test condition. From this result one may say that this characteristic value can become the standard of quantitative definition for "shallow" or "deep" overburden height.

2. A new constructed stress-measuring system was developed for measuring the stress at any location in the model ground. The proposed device is effective to specify the area where the earth pressure changes.

3. The applicability of the superposition of the differences between the isotropic pressure and the overburden pressure at the any horizontal section was examined by introducing the cavity expansion theory for this study. The behavior of increasing the earth pressure around the cavity was expressed very well. However, the some problem on the results with both test and theory exist in the condition of decreasing earth pressure. In next stage, these problems will have to be improved.

\section{Acknowledge}

The authors would like to thank Mr. Kawamura, Mr. Nakashima and Mr. Yamada of Kyushu University for their cooperation in the development of the stress-measuring system.

\section{References}

Kawamura, A. Ochiai, H., Yasufuku, N., Omine, K., Yamada, S. \& Sugimoto, S. (2003): Development and application of stress-measuring system in the aluminum rods stratum, $58^{\text {th }}$ JSCE Annual Meeting, now on contribution (in Japanese).

Sugimoto, S., Ochiai, H., Yasufuku, N., Omine, K. \& Imanishi, H. (2001): Influence of Neighboring Construction of Shield Tunnel on the Existing Structure by Two-Dimensional Model Test, Modern Tunneling Science and Technology, Vol.2 781-786.

Sugimoto, S., Ochiai, H., Yasufuku, N., Omine, K. \& Imanishi, H. (2002): Influence of Shield Tunnel on the Existing Underground and the Circumference Ground, Proc. $2^{\text {nd }}$ Int. Conference on Soil Structure Interaction in Urban Civil Engineering, Vol.2 299-304.

Terzaghi, K. (1943): Theoretical Soil Mechanics, John Wiley \& Sons, New York, 66-75.

Yu, H. (2000): Cavity Expansion Method in Geomechanics, Kluwer Academic Publishers, pp1-94. 